\title{
New Methods of Brain Stimulation: What They Tell Us About the Old Methods and About the Brain
}

\author{
By Jack M. Gorman, MD
}

Electroconvulsive therapy (ECT) is the most effective treatment known for major depression, and it is also effective for treating mania and the tremors of Parkinson's disease. Nevertheless, it is perhaps the most controversial treatment in all of medicine. Opponents accuse ECT of causing "brain damage." The version of ECT shown in the infamous movie One Flew Over the Cuckoo's Nest certainly did not help its image. In the movie, a questionably mentally ill Randle McMurphy is forced to undergo ECT against his will and without anesthesia.

But Hollywood aside, what are the facts? First, as mentioned above, there is no question that ECT works. The vast majority of patients suffering from depression respond. On the other hand, the response is often short-lived, thus relapse rates are high. Sometimes starting antidepressant medication immediately after a successful course of ECT maintains remission, but many patients require repeat courses and some have no choice but to opt for monthly maintenance treatments over the course of years.

In terms of harming the brain, the fact is that memory disturbance during ECT treatment usually resolves within months. Even careful, rigorously conducted neuropsychological test studies have failed to find evidence of permanent memory loss or any other cognitive disturbance. More recently, preclinical studies have shown that electroshock promotes neurogenesis and stimulates the expression in brain of anti-apoptotic molecules like bcl-2. Although the clinical significance of this is unknown, it follows the same pattern as all antidepressants and suggests that far from causing "brain damage," ECT may contribute to the process of neuronal plasticity and survival that is antagonized by stress.

In reality, ECT is an effective but, nevertheless, imperfect treatment of relatively low toxicity. Interestingly, an affluent person in the United States is much more likely to receive ECT for depression than a poor person, quite unusual for something that is truly harmful.

What then accounts for the poor reputation of ECT? One clear reason, but by no means the only one, is the mystery surrounding its mechanism of action. Causing a seizure throughout the brain seems a crude way to deal with the subtle neurochemical and physiological changes that appear to occur in depressed individuals. While modern neuroscience focuses on molecules associated with depression, ECT seems more like "kicking the television" when the picture becomes fuzzy.

For the first time in decades scientists and clinical researchers are testing alternative methods of brain stimulation to treat depression. Transcranial magnetic stimulation (TMS), vagal nerve stimulation (VNS), and deep brain stimulation (DBS) are among them. The latter two are already used in neurologic disorders like refractory epilepsy and Parkinson's disease; their utility in treating depression is still largely unknown. To varying degrees, TMS, VNS, DBS, and other new brain stimulation techniques may be easier to administer than ECT and may produce less memory disturbance. They may also be less expensive. Of course, if they do not work, adverse side effects and practical benefits will be moot. But what may end up being the most important benefits of the new brain stimulation methods could be what they tell us about brain function. Take TMS, for example. The beam of magnetic stimulation can be focused at relatively specific brain areas, something not possible with electrical stimulation used in ECT. High frequency TMS and low frequency TMS have opposite effects on neuronal activity, so that investigators can now either "turn on" or "turn down" specific brain regions and observe the effects both on activity in other brain areas using functional magnetic resonance imaging and on cognition, behavior, and emotion. The famous observations of Penrose on human emotion caused by stimulation of specific brain areas during neurosurgical procedures can now be accomplished non-invasively in normal volunteers and patients with psychiatric illness.

TMS is only one example. Ultimately, the multiple new methods of stimulating the brain all have the potential to reveal new insights into how the brain works. In the meantime, while we wait to see if any of these new methods will have clinical efficacy in treating depression, it is well for us to remember that however mysterious and seemingly crude, ECT is the one method of brain stimulation we have that is proven beyond doubt to relieve depression. CNS 


\section{THE INTERNATIONAL JOURNAL OF \\ Neuropsychiatric Medicine...ONLINE}

\section{CNS Spectrums' CME content is available FREE Online!}

Instant certification upon quiz completion

\section{Simple registration allows easy access to CME sections}

\section{4 hours of FREE CME credit currently available}

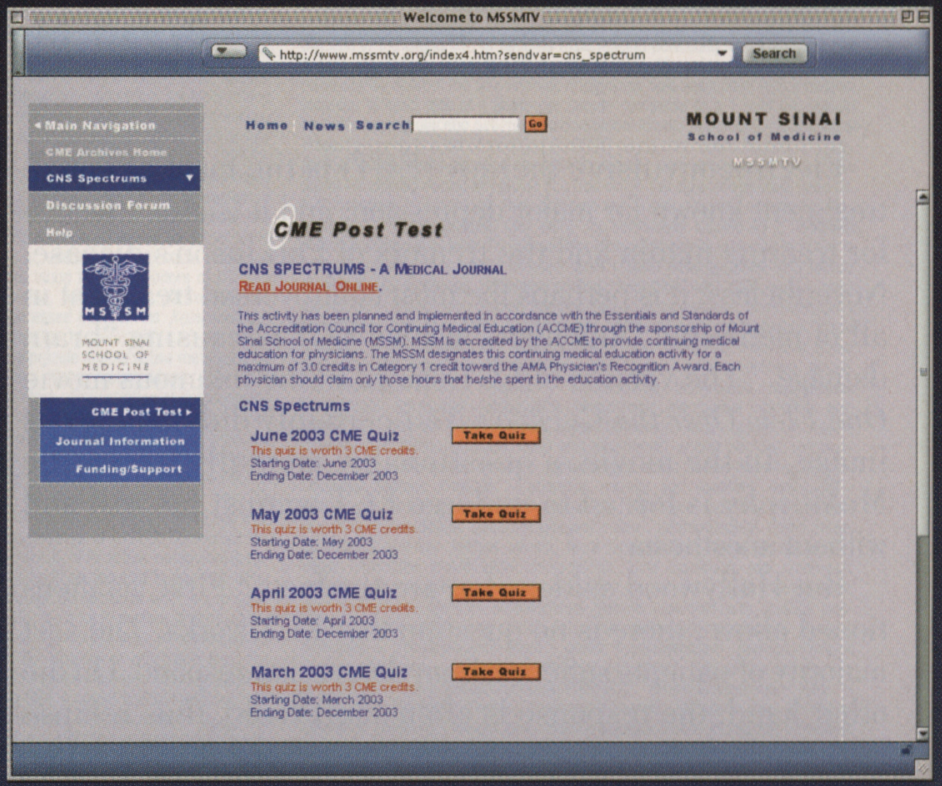

"The International Journal of Neuropsychiatric Medicine" www.cnsspectrums.com

Bipolar Disorder Roundtable Monograph Series An Expert Panel Review of Clinical Challenges in Primary Care and Psychiatry

Earn up to 3 hours of free CME credit by accessing the following expert panel discussions now available online at: www.cnsspectrums.com

Part 1: Recognizing Comorbidities Associated With Bipolar Disorder Moderator: Robert M. A. Hirschfeld, MD

Discussants: Allan H. Young, MB, ChB, and Susan McElroy, MD

Part 2: Remission-Oriented Treatment Considerations in Bipolar Disorder Moderator: Robert M. A. Hirschfeld, MD

Discussants: Michael Gitlin, MD, and Mark Hyman Rapaport, MD

Part 3: Optimiring Therapeutic Options in the Treatment of Bipolar Disorder Moderator: Robert M. A. Hirschfeld, MD

Discussants: David V. Sheehan, MD, MBA, and Martin B. Keller, MD 